\title{
TERPENOS E ÁCIDOS GRAXOS DE Dipteryx lacunifera Ducke
}

\author{
Gerardo Magela Vieira Júnior, Hilris Rocha e Silva, Thaís Chaves Bittencourt e Mariana H. Chaves* \\ Departamento de Química, Universidade Federal do Piauí, 64049-550 Teresina - PI, Brasil \\ Carlos Alberto de Simone \\ Instituto de Química e Biotecnologia, Universidade Federal do Alagoas, 57072-970 Maceió - AL, Brasil
}

Recebido em 6/10/06; aceito em 1/2/07; publicado na web em 28/5/07

\begin{abstract}
TERPENES AND FATTY ACIDS FROM Dipteryx lacunifera Ducke. This paper describes the isolation of the furanocassane-type diterpene, named vinhaticoic acid, along with $\beta$-farnesene and spatulenol from fruit shells of $D$. lacunifera. Structural determinations were accomplished by chemical derivatization and spectral analysis, including 1D and 2D NMR and X-ray crystallography. The fatty portion was extracted from the fruit kernels, transesterfied and analysed by HRGC/MS. Oleic acid (75.8 $\pm 4.3 \%)$ was the major component. Essential oil extracted from the fruit shells of $D$. lacunifera was analysed by HRGC/MS and nine sesquiterpenes were identified; $\beta$-farnesene $(48.6 \%)$ and spatulenol (21.61\%) were the major constituents.
\end{abstract}

Keywords: Dipteryx lacunifera; vinhaticoic acid; fatty acids.

\section{INTRODUÇÃO}

O gênero Dipteryx, pertencente à tribo Dipterygeae, família Leguminosae-Papilionoideae, é constituído por 14 espécies que se destacam por serem utilizadas como plantas medicinais. São encontradas nas regiões amazônica, nordeste e central do Brasil, na Venezuela e na América Central (Costa Rica e Panamá). Algumas frutificam precocemente aos 4 anos, em matas de terras firmes e planas, podendo atingir $50 \mathrm{~m}$ de altura ${ }^{1-4}$.

Quimicamente as espécies do gênero Dipteryx acumulam cumarinas, isoflavonas, triterpenóides, ácidos graxos e diterpenóides furanocassanos ${ }^{2,3,5-9}$. A $D$. odorata é a mais estudada; suas sementes são ricas em cumarinas, importantes na indústria de cosméticos e perfumaria, além de serem comercializadas para distúrbios vasculares e linfáticos ${ }^{10}$ e exibirem atividade farmacológica no sistema muscular ${ }^{2}$. As cascas apresentam cumarinas, lupeol, $\beta$ farneseno, betulina e ésteres metílicos de ácidos $\operatorname{graxos}^{8,9,11}$ e o óleo essencial das flores é rico em germacreno D e espatulenol ${ }^{12}$. Pesquisas recentes, realizadas com as sementes de D. odorata, resultaram no isolamento de vários constituintes químicos, dentre eles um novo diterpenóide furanocassano, uma nova isoflavolignana e a chalcona isoliquiritigenina que exibe um alto potencial preventivo contra câncer em glândulas mamárias de ratos ${ }^{6}$.

Dipteryx lacunifera Ducke, sinonímia Coumarouna lacunifera, conhecida popularmente como castanha de burro e garampara, é encontrada na região meio-norte do Brasil (Maranhão e Piauí) ${ }^{4}$. Esta espécie produz um fruto, cuja amêndoa é comestível, comercializado em feiras livres do município de Bom Jesus-PI, com o nome de fava de morcego. Em trabalho anterior, de Mendes e Silveira ${ }^{2}$, foram identificados ácidos graxos, $\beta$-farneseno e dois diterpenos furanocassanos nos frutos desta espécie.

Este artigo relata pela primeira vez a composição química do óleo essencial das cascas do fruto de D. lacunifera e, adicionalmente, descreve também os constituintes fixos do extrato hexânico das cascas do fruto e do óleo das amêndoas, visando contribuir para o conhecimento da constituição química desta espécie. O estudo fitoquímico do extrato hexânico resultou no isolamento e na identi-

*e-mail: mariana@ufpi.br

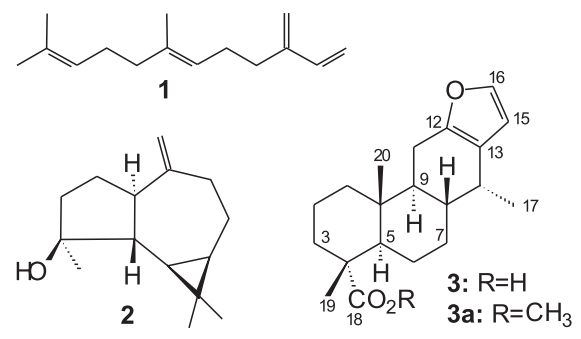

Figura 1. Substâncias isoladas das cascas do fruto de D. lacunifera

ficação de dois sesquiterpenos $\beta$-farneseno (1) e espatulenol (2) e um diterpeno furanocassano, ácido vinhaticóico (3), inédito no gênero Dipteryx (Figura 1). Os sesquiterpenos 1 e 2 também foram identificados no óleo essencial juntamente com sete sesquiterpenos menos abundantes. No óleo fixo das amêndoas, foram identificados cinco ácidos graxos, determinados como ésteres metílicos, sendo o constituinte majoritário o ácido oléico $(75,8 \pm 4,3 \%)$.

\section{PARTE EXPERIMENTAL}

\section{Procedimentos experimentais gerais}

As separações cromatográficas em coluna à pressão atmosférica foram realizadas em gel de sílica $60(0,063-0,20 \mathrm{~mm})$ da Merck e Sephadex LH-20 da Sigma. As placas para cromatografia em camada delgada comparativa (CCDC) foram preparadas com 0,25 $\mathrm{mm}$ de espessura, usando como fase estacionária gel de sílica 60 GF-254 da Merck e $60 \mathrm{G}$ Vetec. As revelações das cromatoplacas foram feitas por borrifamento com solução de sulfato cérico. $\mathrm{O}$ ponto de fusão foi determinado em aparelho da Microquímica, modelo MORF-301, com taxa de aquecimento de $10{ }^{\circ} \mathrm{C} \mathrm{min}^{-1}$ entre 30 e $50{ }^{\circ} \mathrm{C}$ e $1{ }^{\circ} \mathrm{C} \mathrm{min}{ }^{-1}$ até $103{ }^{\circ} \mathrm{C}$. Os espectros de ressonância magnética nuclear (RMN) foram obtidos em espectrômetro Brüker, modelo Avance DRX-500, operando a $500 \mathrm{MHz}$ na freqüência do hidrogênio e a $125 \mathrm{MHz}$ na frequiência do carbono-13. O solvente utilizado na dissolução das amostras para obtenção dos espectros foi o clorofórmio deuterado $\left(\mathrm{CDCl}_{3}\right.$, Isotec - INC).

Os espectros de massas foram obtidos por injeção em 
cromatógrafo a gás acoplado a espectrômetro de massas da Shimadzu, modelo CG-17A e detector de massas modelo QP-5000; voltagem do filamento: $70 \mathrm{eV}$; voltagem do detector: $1,3 \mathrm{KV}$; analisador do tipo quadrupolo. As colunas utilizadas foram DB-1 de $30 \mathrm{~m}$ x $0,25 \mathrm{~mm}$ e $0,25 \mu \mathrm{m}$ de espessura do filme e DB-5 de 50 $\mathrm{m} \times 0,25 \mathrm{~mm}$ e $0,25 \mu \mathrm{m}$ de espessura do filme.

\section{Material vegetal}

O material vegetal (frutos) foi coletado no município de Bom Jesus-PI, em agosto de 2003. A identificação botânica foi realizada pelo Dr. H. C. de Lima do Instituto de Pesquisa Jardim Botânico do Rio de Janeiro. As exsicatas da espécie foram depositadas no Herbário Graziela Barroso-UFPI sob número TEPB 18.246 e no Herbário da Embrapa Meio-Norte sob o número 1704.

\section{Extração e fracionamento}

Os frutos secos foram inicialmente separados em cascas (294 g) e sementes (19 g). As cascas depois de lavadas com hexano, para retirar o excesso de óleo, foram moídas e extraídas por maceração sucessivamente com hexano e etanol, à temperatura ambiente. Após concentração e permanência em dessecador sob vácuo, até peso constante, foram obtidos 20,6 g do extrato hexânico, correspondendo ao rendimento de $7 \%$, calculado em relação ao peso do material vegetal seco. Parte do extrato hexânico $(10 \mathrm{~g})$ foi submetida à cromatografia em coluna de gel de sílica $(38 \times 5,5 \mathrm{~cm}$ - 280 g), preenchida com hexano, com volume morto de $448 \mathrm{~mL}$, utilizando-se como eluente os solventes hexano e acetato de etila, em ordem crescente de polaridade. Foram coletadas 138 frações, de aproximadamente $125 \mathrm{~mL}$, as quais depois de concentradas e analisadas por CCD de gel de sílica foram reunidas em 30 grupos de acordo com a cor observada nos cromatogramas e os fatores de retenção $\left(\mathrm{R}_{\mathrm{f}}\right)$. O grupos A-4 (378 mg) e A-16 (86 mg), eluídos em hexano-AcOEt (95:5), correspondem às substâncias 1 e 2, respectivamente. O grupo A-31 (frações 31-33, $156 \mathrm{mg}$ ), eluído em hexano-AcOEt (95:5), por apresentar em análise por CCD característica de substância ácida (mancha alongada), foi tratado com diazometano. Após filtração em coluna de gel de sílica eluída com hexano-AcOEt (98:2), resultou no isolamento da substância 3a (48 $\mathrm{mg})$. A fração A-34 (602 mg) foi submetida à permeação em coluna de Sephadex LH-20, eluída em $\mathrm{CH}_{2} \mathrm{Cl}_{2}$-acetona (3:2), resultando no isolamento da substância $3(229 \mathrm{mg})$.

$\beta$-Farneseno (1): $\mathrm{C}_{15} \mathrm{H}_{24}$; EMIE $\mathrm{m} / \mathrm{z}(\%) 204$ ([M] $\left.]^{++}, 3\right), 189$ (3), 133 (19), 120 (16), 93 (54), 79 (26), 69 (100), 67 (29), 55 (26), 41 (93). RMN ${ }^{1} \mathrm{H}(500 \mathrm{MHz})$ e ${ }^{13} \mathrm{C}(125 \mathrm{MHz})$, em concordância com os relatados na ref. 13.

Espatulenol (2): $\mathrm{C}_{15} \mathrm{H}_{24} \mathrm{O}$; EMIE $\mathrm{m} / \mathrm{z}(\%) 220\left([\mathrm{M}]^{\cdot+}, 3\right), 205$ (41), 187 (23), 159 (56),145 (30),131 (51),119 (59), 105 (49), 91 (97), 69 (100), 55 (69). RMN ${ }^{1} \mathrm{H}$ (500 MHz) e ${ }^{13} \mathrm{C}$ (125 MHz), em concordância com os relatados na ref. 14 .

Vinhaticoato de metila (3a): $\mathrm{C}_{21} \mathrm{H}_{30} \mathrm{O}_{3}$; cristais incolores; PF 100,8102,$2 ;[\alpha]_{\mathrm{D}}{ }^{20}=+56,3^{\circ}\left(\mathrm{c} 5,5 \times 10^{-4}, \mathrm{CHCl}_{3}\right)$; EMIE $\mathrm{m} / \mathrm{z}(\%) 330\left([\mathrm{M}]^{\circ+}\right.$, 41), 255 (26), 161 (35), 133 (29), 131 (41), 109 (50), 108 (100), 91 (29), 55 (23). RMN ${ }^{1} \mathrm{H}$ (500 MHz) e ${ }^{13} \mathrm{C}$ (125 MHz), Tabela 1.

\section{Dados cristalográficos de 3 a}

Sistema cristalino ortorrômbico e grupo espacial P2 $22_{1} 2_{1}$; $\mathrm{a}=6,8460$ (2) $\AA, \mathrm{b}=8,3980$ (4) $\AA, \mathrm{c}=32,1200$ (4) $\AA$; $\mathrm{V}=1846,67$ (13) $\AA^{3} ; \mathrm{Z}=4 ; \mathrm{D}_{\mathrm{x}}=1,189 \mathrm{~g} \mathrm{~cm}^{-1} ; \lambda\left(\mathrm{MoK}_{\alpha}\right)=0,71013 \AA ; \mathrm{F}(000)=720$; $\mathrm{T}=293 \mathrm{~K}$; cristal incolor com dimensões $0,35 \times 0,19 \times 0,07 \mathrm{~mm}$. Foram coletadas 4061 reflexões e o refinamento foi baseado nos $\mathrm{F}^{2}$, resultando em um índice de discordância $\mathrm{R}_{1}\left[\mathrm{~F}^{2}>2 \sigma\left(\mathrm{F}^{2}\right)\right]=0,066 \mathrm{e}$ $\mathrm{Rw}_{2}=0,121$. No refinamento foram utilizadas 2602 reflexões observadas e foram refinados 217 parâmetros. O parâmetro absoluto de Flack determinado foi $0,92(5)$ e o refinamento do enantiômero resultou em um valor de 1,75(2). As posições dos átomos de hidrogênio ligados aos carbonos foram determinadas através de condições geométricas e seus parâmetros de deslocamentos térmicos foram calculados como 1,5 $\mathrm{U}_{\text {eq }}$ (C-metilas) ou 1,2 $\mathrm{U}_{\text {eq }}$ (outros). A coleta dos dados das intensidades difratadas foi realizada utilizando-se um Difratômetro Automático KappaCCD Mod. FR590 da Enraf-Nonius. Os programas utilizados no estudo cristalográfico foram: coleta dos dados, COLLECT ${ }^{15}$; determinação e refinamento da cela unitária, DENZO $^{16}$ e COLLECT; redução dos dados, DENZO e COLLECT; programa utilizado para resolver a estrutura, SHELXS97 ${ }^{17}$; programa utilizado para refinar a estrutura, SHELXL97 ${ }^{17}$; programa para elaboração do gráfico molecular, ORTEP- $3^{18}$; o software utilizado na preparação do material para a publicação foi o WinGX-Routine ${ }^{19}$. Dados cristalográficos suplementares foram depositados no Cambridge Crystallographic Data Centre com número de referência CCDC 618652. As cópias do material disponível podem ser obtidas, livre da carga em: CCDC, 12 Union Road, Cambridge CH2 1EZ, UK (fax: +44-1223-336-033; e-mail: deposit@ccdc.cam.ac.uk ou http://www.ccdc.cam.ac.uk).

\section{Extração e análise do óleo essencial}

As cascas do fruto (336 g) foram submetidas à hidrodestilação, por um período de $3 \mathrm{~h}$, em aparelhagem Cleavenger modificada. $\mathrm{O}$ óleo foi coletado em éter etílico ( $3 \mathrm{~mL}$ ) e após a evaporação do solvente foram obtidos $2,1 \mathrm{~g}$ de óleo essencial $(0,62 \%$ de rendimento). O óleo essencial foi analisado por CGAR/EM, com coinjeção de padrões de hidrocarbonetos $\left(\mathrm{C}_{10} \mathrm{a}_{30}\right)$, utilizando coluna capilar de sílica fundida (DB-1), temperatura programada da coluna: $75^{\circ} \mathrm{C}$ por $8 \mathrm{~min}$, taxa de aquecimento $6^{\circ} \mathrm{C} \mathrm{min}^{-1}$ até $200^{\circ} \mathrm{C}$ mantido por $4 \mathrm{~min}$; temperatura do injetor: $150^{\circ} \mathrm{C}$; temperatura da interface: $280{ }^{\circ} \mathrm{C}$; gás de arraste: hélio, com fluxo de $0,5 \mathrm{~mL} \mathrm{~min}{ }^{-1}$. A corrida com detector de massas foi feita no modo scan, com tempo de aquisição de $47 \mathrm{~min}$ e corte do solvente em $8 \mathrm{~min}$; faixa de massas: 50 a 350 daltons. Os compostos foram identificados também por comparação com os espectros de massas da Biblioteca Wiley 229.

\section{Fracionamento do óleo essencial}

Parte do óleo essencial $(1,9 \mathrm{~g})$ foi submetido à cromatografia em coluna de gel de sílica (55 g), preenchida com hexano, com volume morto de $88 \mathrm{~mL}$, utilizando como eluente éter de petróleo (100\%) e $\mathrm{CH}_{2} \mathrm{Cl}_{2}$ (100\%). Foram coletadas 15 frações, de aproximadamente $45 \mathrm{~mL}$, as quais depois de concentradas e analisadas por CCD de gel de sílica foram reunidas em três grupos, de acordo com a cor revelada nos cromatogramas e os $\mathrm{R}_{\mathrm{f}}$. Os grupos OE-1 (17, 1,4 g) e OE-11 (11-14, $63 \mathrm{mg})$ correspondem, após analise por RMN, à substância 1 e à mistura contendo 2 como substância majoritária, respectivamente.

\section{Extração do óleo fixo}

As amêndoas $(6,2 \mathrm{~g})$ foram trituradas, transferidas para cartucho de um aparelho extrator Soxhlet e extraídas com hexano, em triplicata, durante $6 \mathrm{~h}$. Após evaporação do solvente, o óleo foi colocado em dessecador até atingir peso constante ${ }^{20,21}$. O percentual de óleo foi calculado sobre o peso da amostra seca, obtendo-se um teor de lipídios de 39,25 $\pm 2,0 \%$. 
Tabela 1. Dados de RMN ${ }^{1} \mathrm{H}(500 \mathrm{MHz})$ e ${ }^{13} \mathrm{C}(125 \mathrm{MHz})$ das substâncias 3 e 3a, incluindo resultados de mapas de contornos de correlação heteronuclear ${ }^{1} \mathrm{H}^{-13} \mathrm{C}-\mathrm{COSY}{ }^{-n} \mathrm{~J}_{\mathrm{CH}}(\mathrm{n}=1, \mathrm{HMQC} ; \mathrm{n}=2$ e $3, \mathrm{HMBC})$, em $\mathrm{CDCl}_{3}$. Deslocamentos químicos em $\delta$ (ppm) e constantes de acoplamento ( $J$, entre parênteses) em $\mathrm{Hz}^{*}$

\begin{tabular}{|c|c|c|c|c|c|c|c|c|}
\hline & \multicolumn{4}{|c|}{3} & \multicolumn{4}{|c|}{$3 \mathbf{a}$} \\
\hline & \multicolumn{2}{|r|}{ HMQC } & \multicolumn{2}{|c|}{ HMBC } & \multicolumn{2}{|r|}{ HMQC } & \multicolumn{2}{|c|}{ HMBC } \\
\hline & $\delta_{\mathrm{C}}$ & $\delta_{\mathrm{H}}$ & ${ }^{2} J_{\mathrm{CH}}$ & ${ }^{3} J_{\mathrm{CH}}$ & $\delta_{\mathrm{C}}$ & $\delta_{\mathrm{H}}$ & ${ }^{2} J_{\mathrm{CH}}$ & ${ }^{3} J_{\mathrm{CH}}$ \\
\hline \multicolumn{9}{|l|}{ C } \\
\hline 4 & 47,5 & - & Н-5; 3Н-19 & - & 47,4 & - & $\begin{array}{c}2 \mathrm{H}-3 ; \mathrm{H}-5 ; \\
3 \mathrm{H}-19\end{array}$ & $2 \mathrm{H}-2$ \\
\hline 10 & 37,2 & - & H-5; 3H-20 & $\mathrm{H}-8$ & 36,8 & - & $\begin{array}{l}2 \mathrm{H}-1 ; \mathrm{H}-5 ; \\
\mathrm{H}-9 ; 3 \mathrm{H}-20\end{array}$ & $\begin{array}{c}2 \mathrm{H}-2 ; \mathrm{H}-8 ; \\
2 \mathrm{H}-11\end{array}$ \\
\hline 12 & 149,2 & - & $2 \mathrm{H}-11$ & $\begin{array}{c}\text { H-14; H-15; } \\
\text { H-16 }\end{array}$ & 149,4 & - & 2H-11 & $\begin{array}{c}\text { H-14; H-15; } \\
\text { H-16 }\end{array}$ \\
\hline 13 & 122,4 & - & H-14; H-15 & $\begin{array}{c}2 \mathrm{H}-11 ; \\
\mathrm{H}-16 ; 3 \mathrm{H}-17\end{array}$ & 122,4 & - & Н-14; H-15 & $\begin{array}{c}2 \mathrm{H}-11 ; \mathrm{H}-16 ; \\
3 \mathrm{H}-17\end{array}$ \\
\hline 18 & 184,9 & - & - & 3Н-19 & 179,3 & - & - & $\begin{array}{c}\mathrm{H}-5 ; 3 \mathrm{H}-19 ; \\
\mathrm{O}-\mathrm{CH}_{3}\end{array}$ \\
\hline \multicolumn{9}{|l|}{$\mathrm{CH}$} \\
\hline 5 & 49,5 & $1,79(m)$ & - & $\begin{array}{l}\mathrm{H}-9 ; 3 \mathrm{H}-19 ; \\
3 \mathrm{H}-20\end{array}$ & 49,6 & $1,75(m)$ & H-6 & $\begin{array}{c}2 \mathrm{H}-1 ; 2 \mathrm{H}-3 ; \\
2 \mathrm{H}-7 ; \mathrm{H}-9 ; \\
3 \mathrm{H}-19 ; 3 \mathrm{H}-20\end{array}$ \\
\hline 8 & 36,0 & $1,77(m)$ & $\mathrm{H}-14$ & 3H-17 & 35,6 & $1,75(m)$ & H-9; H-14 & H-9; H-14 \\
\hline 9 & 46,0 & $1,66(m)$ & - & H-5; 3H-20 & 45,6 & $1,64(m)$ & 2H-11 & $\begin{array}{l}\mathrm{H}-5 ; 2 \mathrm{H}-7 ; \\
\mathrm{H}-14 ; 3 \mathrm{H}-20\end{array}$ \\
\hline 14 & 31,8 & 2,58 (quint, 7,0) & H-8; 3H-17 & - & 31,4 & 2,59 (quint, 7,0 ) & $3 \mathrm{H}-17$ & 2H-7; H-9 \\
\hline 15 & 109,6 & $6,19(d, 1,6)$ & H-16 & $\mathrm{H}-14$ & 109,5 & $6,19(d, 1,5)$ & H-16 & H-14 \\
\hline 16 & 140,3 & $7,23(d, 1,1)$ & $\mathrm{H}-15$ & - & 140,3 & $7,23(s l)$ & H-15 & - \\
\hline \multicolumn{9}{|l|}{$\mathrm{CH}_{2}$} \\
\hline 1 & 37,1 & 1,68 e 1,79 & $2 \mathrm{H}-2$ & $\begin{array}{l}\text { H-5; H-9; } \\
3 \mathrm{H}-20\end{array}$ & 36,7 & 1,62 e 1,75 & $2 \mathrm{H}-2$ & $\begin{array}{l}2 \mathrm{H}-3 ; \mathrm{H}-5 ; \\
\mathrm{H}-9 ; 3 \mathrm{H}-20\end{array}$ \\
\hline 2 & 18,2 & $1,52-1,69$ & $2 \mathrm{H}-3$ & - & 17,8 & $1,50-1,68$ & $2 \mathrm{H}-1 ; 2 \mathrm{H}-3$ & - \\
\hline 3 & 38,8 & 1,16 e 1,70 & - & - & 38,5 & 1,15 e 1,68 & $2 \mathrm{H}-2$ & $\begin{array}{c}2 \mathrm{H}-1 ; \mathrm{H}-5 ; \\
3 \mathrm{H}-19\end{array}$ \\
\hline 6 & 31,1 & 1,49 e 1,69 & $\mathrm{H}-5$ & $\mathrm{H}-8$ & 30,7 & 1,48 e 1,68 & $\mathrm{H}-5 ; 2 \mathrm{H}-7$ & H-8 \\
\hline 7 & 24,4 & 1,30 e 1,50 & $\mathrm{H}-8$ & $\mathrm{H}-5$ & 24,0 & 1,20 e 1,47 & $2 \mathrm{H}-6$ & $\mathrm{H}-5$ \\
\hline 11 & 22,4 & $\begin{array}{c}2,39(d d, 10,2 \text { e } 16,8) \\
2,59(d d, 6,9 \text { e } 16,8)\end{array}$ & H-9 & - & 21,9 & $\begin{array}{c}2,40(d d, 10,2 \text { e } 16,8) \\
2,60(d d, 6,9 \text { e } 16,8)\end{array}$ & H-9 & - \\
\hline $\begin{array}{l}\mathrm{CH}_{3} \\
17\end{array}$ & 18,0 & $1,00(d, 7,0)$ & - & $\mathrm{H}-8$ & 17,5 & $1,00(d, 7,0)$ & $\mathrm{H}-14$ & - \\
\hline 19 & 17,1 & $1,24(s)$ & - & H-5 & 17,0 & $1,24(s)$ & - & H-5 \\
\hline 20 & 15,0 & $0,96(s)$ & - & $2 \mathrm{H}-1 ; \mathrm{H}-5 ; \mathrm{H}-9$ & 14,6 & $0,94(s)$ & - & $2 \mathrm{H}-1 ; \mathrm{H}-5$ \\
\hline $\mathrm{CH}_{3} \mathrm{O}$ & - & - & - & - & 51,8 & $3,69(s)$ & - & - \\
\hline
\end{tabular}

*Análise comparativa do espectro de RMN ${ }^{13} \mathrm{C}$-DEPT foi usada para reconhecer os átomos de carbono não hidrogenados, metínicos, metilênicos e metílicos. Deslocamentos químicos e constantes de acoplamento $(J)$ dos átomos de hidrogênio foram deduzidos dos espectros 1D de RMN ${ }^{1} \mathrm{H}$ e dos mapas de contornos $2 \mathrm{D}{ }^{1} \mathrm{H}-{ }^{1} \mathrm{H}-\mathrm{COSY}$ e ${ }^{1} \mathrm{H}-{ }^{13} \mathrm{C}-\mathrm{COSY}-\mathrm{HMQC}$. Sinais de átomos de hidrogênio sem multiplicidade indicam sobreposição.

\section{Transesterificação do óleo fixo e análise dos ésteres metílicos}

Os triacilgliceróis do óleo foram transesterificados, seguindo metodologia IUPAC ${ }^{22}$, com algumas modificações, por meio do seguinte procedimento: em tubo de ensaio, foram adicionados cerca de $100 \mathrm{mg}$ do óleo, $2 \mathrm{~mL}$ de hexano e $0,2 \mathrm{~mL}$ de solução metanólica $2 \mathrm{~mol} \mathrm{~L}^{-1}$ de $\mathrm{KOH}$. O tubo de ensaio foi agitado por $5 \mathrm{~min}$ em agitador vortex e foram adicionados $2 \mathrm{~mL}$ de solução saturada de cloreto de sódio, até a separação da fase orgânica ${ }^{21-23}$. Os ésteres metílicos dos ácidos graxos foram analisados em triplicata, após injeção de 1 $\mu \mathrm{L}$ da fase orgânica em cromatógrafo a gás acoplado a espectrômetro de massas, utilizando coluna capilar DB-5. As condições de operação foram: temperatura programada da coluna: $150{ }^{\circ} \mathrm{C}$ por $2 \mathrm{~min}$; velocidade de aquecimento $5{ }^{\circ} \mathrm{C} / \mathrm{min}$ até $230{ }^{\circ} \mathrm{C}$ por $7 \mathrm{~min}$; velocidade de aquecimento $4{ }^{\circ} \mathrm{C} / \mathrm{min}$ até $260{ }^{\circ} \mathrm{C}$ mantida por 7,5 min; temperatura do injetor: $230{ }^{\circ} \mathrm{C}$; temperatura da interface: $260{ }^{\circ} \mathrm{C}$; gás de arraste: hélio, com fluxo de $1,0 \mathrm{~mL} / \mathrm{min}$. A corrida com detector de massa foi feita no modo scan, com tempo de aquisição de $32 \mathrm{~min}$ e corte do solvente em 8 min; faixa de massas: 40 a 450 Daltons; voltagem do filamento: $70 \mathrm{eV}$; voltagem do detector: $1,3 \mathrm{KV}$; analisador do tipo quadrupolo. Os ésteres metílicos de ácidos graxos foram identificados por similaridade (maiores índices de similaridades - IS) dos espectros de massas dos picos do cromatograma de íons totais obtido com os da biblioteca do equipamento.

\section{RESULTADOS E DISCUSSÃO}

As cascas do fruto de D. lacunifera, ricas em um óleo viscoso de odor forte e relativamente agradável, forneceram o extrato hexânico, do qual foram isoladas as substâncias 1-3. 
A identificação de $\mathbf{1}$ e $\mathbf{2}$ como sendo $\beta$-farneseno e espatulenol, respectivamente, foi feita por meio da análise dos seus espectros de massas, RMN ${ }^{1} \mathrm{H}$ e ${ }^{13} \mathrm{C}$ e comparação com dados da literatura ${ }^{13,14}$.

A substância 3 corresponde ao ácido vinhaticóico. $\mathrm{O}$ espectro de RMN ${ }^{1} \mathrm{H}$ de 3 apresentou, entre outros, dois dupletos em $\delta$ 6,19 $(J=1,6$ Hz, H-15) e 7,23 ( $J=1,1 \mathrm{~Hz}, \mathrm{H}-16)$, característicos de hidrogênios em anel furânico ${ }^{24,25}$. Além destes, apresentou dois simpletos em $\delta$ 0,96 e 1,24 e um dupleto em $\delta 1,00(J=7,0 \mathrm{~Hz})$ correspondentes a hidrogênios de grupos metílicos, atribuídos a H-20, H-19 e H-17, respectivamente. $\mathrm{O}$ espectro de $\mathrm{RMN}{ }^{13} \mathrm{C}-\mathrm{BB}{ }^{1} \mathrm{HD}$ de 3 (Tabela 1) apresentou 20 sinais e sua análise conjunta com o espectro de RMN ${ }^{13} \mathrm{C}$-DEPT $135^{\circ}$ permitiu identificar a presença de sinais referentes a três carbonos metílicos, seis metilênicos, seis metínicos e cinco não hidrogenados, entre os quais um em $\delta 184,9$ relativo a carbonila de grupamento ácido e dois pares de sinais em $\delta 122,4$ (C) e 149,2 (C); $\delta$ 109,6 $(\mathrm{CH})$ e 140,3 $(\mathrm{CH})$ relativos aos carbonos do anel furânico.

$\mathrm{A}$ análise dos dados de $\mathrm{RMN}{ }^{13} \mathrm{C}$ permitiu atribuir à substância 3 a fórmula molecular $\mathrm{C}_{20} \mathrm{H}_{28} \mathrm{O}_{3}$, com equivalente de insaturação igual a sete, sendo consistente com a presença do anel furânico, uma carbonila e, portanto, três anéis.

O tratamento de $\mathbf{3}$ com diazometano conduziu à formação de 3a, cuja reação foi confirmada pela observação, no espectro de $\mathrm{RMN}{ }^{1} \mathrm{H}$, do simpleto em $\delta 3,69$ correspondente a grupo metoxílico. Os dados de RMN ${ }^{13} \mathrm{C}$-BB ${ }^{1} \mathrm{HD}$ e DEPT $135^{\circ}$ de 3a mantiveram a coerência esperada em relação à substância 3. Observou-se um sinal adicional em $\delta_{\mathrm{C}} 51,8$ correspondente à metoxila e que o sinal da carbonila foi deslocado para $\delta_{\mathrm{C}} 179,3$, caracterizando o éster ${ }^{25,26}$. Os dados de RMN observados para 3a foram semelhantes aos registrados na literatura para o vinhaticoato de metila ${ }^{24,27}$. Os mapas de contornos HMQC e HMBC possibilitaram atribuir os deslocamentos químicos do ácido vinhaticóico (3) e do seu derivado metilado, vinhaticoato de metila (3a), Tabela 1.

A definição da configuração relativa do carbono, C-4 com o grupo carboxila (C-18) em posição equatorial (cis) em relação a $\mathrm{H}-5$ dos diterpenos $\mathbf{3}$ e $\mathbf{3 a}$, baseou-se no deslocamento químico dos sinais de C-5, C-4 e C-19 ${ }^{28}$. Observou-se que o sinal de C-5 aparece em $\delta 49,5$, porém se o grupo carboxílico (ou COOMe) estivesse em posição axial (trans) o deslocamento químico de C-5 seria de aproximadamente $\delta 56,0$. Conseqüentemente, o carbono metílico (C-19) ocupa a posição axial e seu deslocamento químico observado é $\delta 17,1$, pois em posição equatorial este seria de aproximadamente $\delta 29,0^{28}$. Além disso, o sinal de C-4 exibe um deslocamento químico em $\delta$ 47,5 e se grupo carboxílico (ou COOMe) estivesse em orientação axial seria de $\delta 43,0^{28}$.

Todos os dados espectroscópicos estão em concordância com as estruturas propostas para $\mathbf{3}$ e $\mathbf{3 a}$, com a estereoquímica relativa de C4 confirmada por cristalografia de raio $\mathrm{X}$ (Figura 2). No entanto, os valores obtidos para o parâmetro de Flack, o qual deveria ser 0,0 para o enantiômero correto, não permitiram determinar a configuração absoluta da molécula ${ }^{29}$. Desta forma, a estereoquímica absoluta de $\mathbf{3}$ foi sugerida com base no sinal positivo do desvio do plano da luz polarizada, caracterizando o diterpeno como pertencente à série normal $\left(\mathrm{H}-5 \alpha, \mathrm{CH}_{3}-20 \beta\right)^{30}$, a exemplo de outros diterpenóides furanocassanos da série normal, com a estereoquímica absoluta determinada por cristalografia de raio $\mathrm{X}$, tais como ácido dipteríxico $\left([\alpha]_{D}+64^{\circ}\right)^{6}$, Caesalmina $\mathrm{C}\left([\alpha]_{\mathrm{D}}+51,2^{\circ}\right)^{31}$, Caesalmina $\mathrm{D}\left([\alpha]_{\mathrm{D}}\right.$ $\left.+65,9^{\circ}\right)^{30}$ e Taepeenina A $\left([\alpha]_{D}+56,6^{\circ}\right)^{32}$.

Este é o primeiro relato da ocorrência do ácido vinhaticóico no gênero Dipteryx (Leguminosae-Papilionoideae), tendo sido anteriormente isolado apenas em Caesalpinia crista (LeguminosaeCaesalpinioideae) ${ }^{32}$. Seu derivado metilado, vinhaticoato de metila, encontra-se descrito em Caesalpinia crista $^{32}$, em Plathymenia

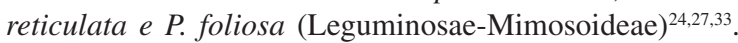

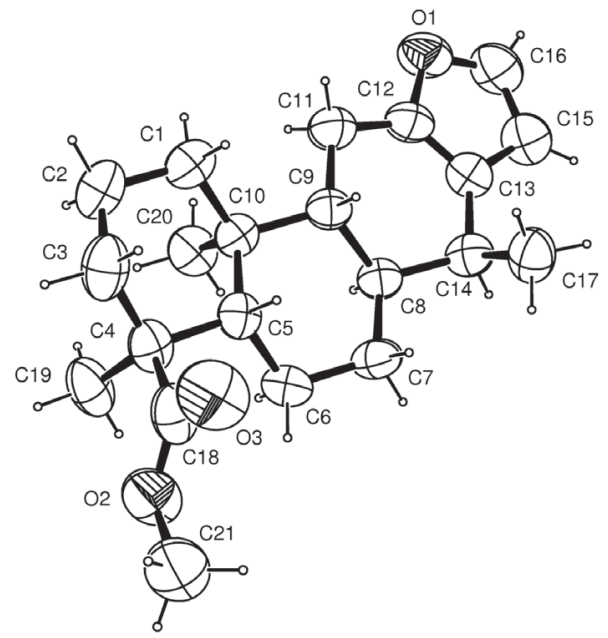

Figura 2. Projeção ORTEP-3 de 3 a com os átomos identificados e elipsóides de vibração térmica a $50 \%$ de probabilidade

\section{Óleo essencial}

A análise do óleo essencial de D. lacunifera por CGAR/EM apresentou um cromatograma de íons totais correspondentes a nove sesquiterpenos (Tabela 2), sendo o $\beta$-farneseno e espatulenol os componentes majoritários. O óleo essencial bruto foi analisado por RMN ${ }^{1} \mathrm{H}$ e ${ }^{13} \mathrm{C}$, confirmando a identificação do sesquiterpeno $\beta$ farneseno como o componente mais abundante.

Tabela 2. Composição química do óleo essencial, tempo de retenção (Tr), Índice de Kovatz da literatura (IKL), calculado (IKC) e porcentagem $(\%)$

\begin{tabular}{lcccc}
\hline Substância & $\begin{array}{c}\mathrm{Tr} \\
(\mathrm{min})\end{array}$ & $\mathrm{IKL}^{35}$ & IKC & $\begin{array}{c}\text { Porcentagem } \\
(\%)\end{array}$ \\
\hline$\alpha$-Copaeno & 21,067 & 1376 & 1377 & 2,33 \\
$\beta$-Elemeno & 21,367 & 1391 & 1389 & 0,68 \\
trans-Cariofileno & 22,067 & 1418 & 1419 & 5,74 \\
$\beta$-Farneseno* & 22,667 & 1443 & 1445 & 27,82 \\
Germacreno-D & 23,500 & 1480 & 1481 & 3,04 \\
Biciclogermacreno $^{2}$ & 23,825 & 1494 & 1495 & 3,26 \\
-Cadineno $_{\text {Espatulenol* }}^{24,192}$ & 1513 & 1512 & 0,92 \\
Óxido de cariofileno* & 25,258 & 1576 & 1562 & 13,32 \\
\hline Total & 25,400 & 1581 & 1568 & 1,18 \\
\hline
\end{tabular}

*Identidade confirmada através de análise por RMN, após separação cromatográfica.

Para confirmação da identidade de outros constituintes, o óleo essencial foi submetido à separação cromatográfica resultando em três grupos de frações, dentre estes, $\mathrm{OE}-1$ (1,4 g) corresponde ao $\beta$-farneseno e OE-11 (63 mg) a uma mistura de espatulenol, como constituinte predominante e, entre outros, o óxido de cariofileno, identificados por RMN ${ }^{13} \mathrm{C}$ e comparação com dados da literatura ${ }^{13,34}$.

\section{Ácidos graxos}

O produto obtido da transesterificação dos triacilgliceróis presentes no óleo das amêndoas apresentou na análise por CGAR/EM um cromatograma de íons totais, no qual se observa a presença de nove picos, cuja comparação dos espectros correspondentes com os da biblioteca do aparelho permitiu identificar cinco ésteres 
metílicos derivados de ácidos graxos (Tabela 3). Verificou-se que 77,6\% dos ésteres metílicos identificados são derivados de ácidos graxos insaturados. O teor de ácidos graxos insaturados do óleo das amêndoas de $D$. lacunifera foi superior aos saturados em concordância com o descrito na literatura ${ }^{2}$.

Tabela 3. Composição dos ácidos graxos do óleo das amêndoas de D. lacunifera (\% p/p de ésteres metílicos)

\begin{tabular}{lcr}
\hline Ácido graxo & $\operatorname{Tr}(\mathrm{min})$ & \multicolumn{1}{c}{$\% \pm \mathrm{DP}$} \\
\hline Palmítico, C16:0 & 15,9 & $14,34 \pm 4,61$ \\
Linoléico, C18:2 & 19,2 & $1,76 \pm 0,92$ \\
Oléico, C18:1 cis 9 & 19,3 & $75,82 \pm 4,31$ \\
Esteárico, C18:0 & 19,7 & $4,55 \pm 0,43$ \\
Lignocérico, C24:0 & 30,9 & $3,51 \pm 0,39$
\end{tabular}

Total

100

$\mathrm{DP}=$ desvio padrão

\section{CONCLUSÃO}

O estudo químico do extrato hexânico das cascas do fruto da espécie $D$. lacunifera, permitiu isolar e identificar três constituintes, sendo dois sesquiterpenos, $\beta$-farneseno e espatulenol, e um diterpenóide de esqueleto cassano denominado ácido vinhaticóico, inédito no gênero Dipteryx.

O óleo essencial das cascas do fruto, cuja composição química está sendo relatada pela primeira vez, mostrou-se constituído predominantemente por $\beta$-farneseno. Além deste, foram identificados mais oito sesquiterpenos: $\alpha$-copaeno, $\beta$-elemeno, trans-cariofileno, germacreno-D, biciclogermacreno, $\gamma$-cadineno, espatulenol e óxido de cariofileno. Após fracionamento do óleo essencial, foi isolado o $\beta$-farneseno e uma mistura de sesquiterpenóides oxigenados, com predominância do espatulenol e, entre outros, o óxido de cariofileno.

A composição dos ácidos graxos dos triacilgliceróis do óleo fixo das amêndoas, determinada como ésteres metílicos, apresentou o ácido oléico como o componente majoritário.

\section{AGRADECIMENTOS}

Ao Dr. E. C. E. Araújo da Embrapa Meio Norte-PI pelo fornecimento do material vegetal, ao Dr. H. C. de Lima do Instituto de Pesquisa Jardim Botânico do Rio de Janeiro pela identificação, aos órgãos de fomento pela bolsa de Iniciação Científica e de Mestrado concedidas ao aluno G. M. Vieira Jr. e M. H. Chaves (CNPq) e pelo apoio financeiro (CNPq, CAPES/PROCAD e FINEP), ao Centro Nordestino de Aplicação e Uso da Ressonância Magnética Nuclear (CENAUREMN) da UFC pela obtenção dos espectros de RMN e ao LAPETRO-UFPI pelos espectros de massas.

\section{REFERÊNCIAS}

1. Ducke, A.; An. Acad. Brasil. Cienc. 1948, 1, 39.

2. Mendes, F. N. P.; Silveira, E. R.; Phytochemistry 1994, 35, 1499.

3. Godoy, R. L. O.; Lima, P. D. D. B.; Pinto, A. C.; Neto, F. R. A.; Phytochemistry 1989, 28, 642.

4. http://www.ildis.org/LegumeWeb, acessada em Agosto 2006.

5. Januário, A. H.; Lourenço, M. V.; Domézio, L. A.; Pietro, R. C. L. R.; Castilho, M. S.; Tomazela, D. M.; Silva, M. F. G. F.; Vieira, P. C.; Fernandes, J. B.; França, S. C.; Chem. Pharm. Bull. 2005, 53, 740.

6. Jang, D. S; Park, E. J.; Hawthorne, M. E.; Vigo, J. S.; Graham, J. G.; Cabieses, F.; Santarsiero, B. D.; Mesecar, A. D.; Fong, H. H. S.; Mehta, R. G.; Pezzuto, J. M.; Kinghorn, A. D.; J. Nat. Prod. 2003, 66, 584.

7. Sullivan, G.; J. Agric. Food Chem. 1982, 30, 609.

8. Nakato, T.; Suarez, M.; Planta Med. 1970, 18, 79.

9. Nakato, T.; Alonso, J.; Grillet, R.; Martin, A.; J. Chem. Soc., Perkin Trans. 1 1979, 9, 2107.

10. Bessa, D. T. O.; Mendonça, M. S.; Araújo, M. G. P.; Acta Amazônica 2001, $31,357$.

11. Hayashi, T.; Thomson, R. H.; Phytochemistry 1974, 13, 1943.

12. Andrade, E. H. A.; Zoghbi, M. G. B.; Carreira, L. M. M.; Maia, J. G. S.; J. Essent. Oil Res. 2003, 15, 211.

13. Rahman, A. U.; Ahmad, V. U.; ${ }^{13} C-N M R$ of Natural Products: monoterpenes and sesquiterpenes, Plenum Press: New York, 1992, vol.1.

14. Chaves, M. H.; Tese de Doutorado, Universidade de São Paulo, Brasil, 1996.

15. Enraf-Nonius. COLLECT. Nonius BV, Delft, Holanda, 1997-2000.

16. Otwinowski, Z.; Minor, W. Em Methods in Enzymology; Carter Jr., C. W.; Sweet, R. M., eds.; Academic Press: New York, 1997, p. 307.

17. Sheldrick, G. M.; SHELXS97 and SHELXL97, Universidade de Göttingen, Alemanha, 1997.

18. Farrugia, L. J. J.; Appl. Cryst. 1997, 30, 565

19. Farrugia, L. J. J.; Appl. Cryst. 1999, 32, 837.

20. Instituto Adolfo Lutz; Normas Analíticas do Instituto Adolfo Lutz. Métodos químicos e físicos para análise de alimentos, $3^{a}$ ed., São Paulo, 1985, vol. 1, p. 25,42 e 245 .

21. Chaves, M. H.; Barbosa, A. S.; Moita Neto, J. M.; Aued-Pimentel, S.; Lago, J. H. G.; Quim. Nova 2004, 27, 404.

22. IUPAC; Standard Methods for Analysis of Oils, Fats and Derivatives, Blackwell Scientific Plubications, $7^{\text {th }}$ ed., 1987, IUPAC Method 2.301, Report of IUPAC Working Group WG 2/87.

23. Aued-Pimentel, S.; Lago, J. H. G.; Chaves, M. H.; Kumagai, E. E.; J. Chromatogr., A 2004, 1054, 235.

24. Matos, F. J. A.; Craveiro, A. A.; Maurera, M. A. M. A.; J. Nat. Prod. 1984, 47,581 .

25. Silverstein, R. M.; Webster, F. X.; Identificação Espectrométrica de Compostos Orgânicos, $6^{\mathrm{a}}$ ed., LTC: Rio de Janeiro, 2000.

26. Vieira-Jr. G. M.; Souza, C. M. L.; Chaves, M. H.; Quim. Nova 2005, $28,183$.

27. Leal, R. S.; Lima, M. A. S.; Silveira, E. R.; J. Braz. Chem. Soc. 2003, 14, 120.

28. Velandia, J. R.; Carvalho, M. G.; Braz-Filho, R.; Quim. Nova 1998, 21, 397.

29. Flack, H. D.; Bernardinelli, G.; J. Appl. Crystallogr. 2000, 33, 1143.

30. Dewick, P. M.; Medicinal Natural Products: a biosynthetic approach, $2^{\text {nd }}$ ed., John Wiley \& Sons: New York. 2002.

31. Jiang, R.-W.; Ma, S.-C.; But, P. P.-H.; Mak, T. C. W.; J. Nat. Prod. 2001, $64,1266$.

32. Cheenpracha, S.; Srisuwan, R.; Karalai, C.; Ponglimanont, C.; Chantrapromma, S.; Chantrapromma, K.; Fun, H-K.; Anjum, S.; Rahman, A. U.; Tetrahedron 2005, 61, 8656. Em Narayanan, C. R.; Venkatasubramanian, N. K.; Tetrahedron Lett. 1965, 41, 3639.

33. King, F. E.; King, T. J.; Neill, K. G.; J. Chem. Soc. 1953, 1055.

34. Chaves, M. H.; Brochini, C. B.; Núñez, C. V.; Moreira, I. C.; Roque, N. F.; Martins, D.; Quim. Nova 1999, 22, 37.

35. Adams, R. P.; Identification of essential oil components by gas chromatography/mass spectroscopy, Allured Publishing Corporation, Carol Stream: Illinois, 1995 